\section{Treatment of a pancreatic endocrine tumor by ethanol injection guided by endoscopic ultrasound}
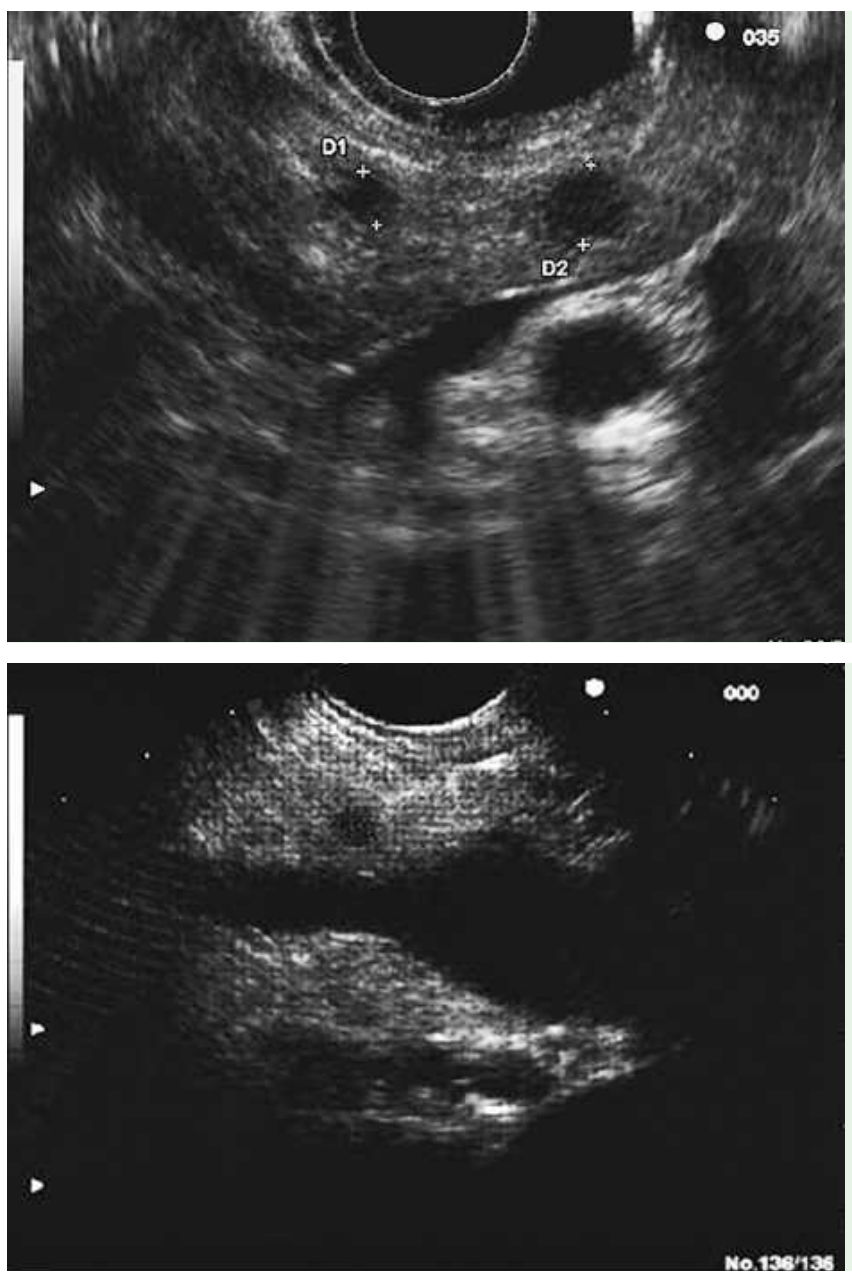

We report the case of a female patient with double endocrine neoplasia type 1 . Echoendoscopy (EUS) revealed two nodular hypoechoic lesions ( $\bullet$ Fig. 1), with a color Doppler hypervascular pattern located in the pancreas. We carried out an EUS-guided fine-needle aspiration (FNA) biopsy [1] (๑ Fig. 2), which showed a neuroendocrine islet tumor in the pathology report 2 weeks later. Tumor sizes were 11 and $7 \mathrm{~mm}$ respectively.
Fig. 1 Echoendoscopy revealed two nodular hypoechoic lesions.

Fig. 2 Endoscopic ultrasound-guided fineneedle aspiration of the pancreatic lesion showed a neuroendocrine islet tumor.
Because the patient refused surgical therapy, we performed an ethanol injection under EUS guidance into the nodule. Ethanol concentration was $40 \%$ [2] and its amount did not exeed $2 \mathrm{ml}$. At the same time celiac plexus neurolysis was carried out to control abdominal pain.

Clinical, biochemical, and transabdominal ultrasonography controls after the EUS-guided injection session and antibiotic treatment were well tolerated, with- out adverse effects. The patient was discharged from hospital 5 days later.

After the first injection, the patient underwent a second session following the same procedure. Unfortunately, during needle retraction, there was minimal ethanol effusion that caused a small pancreatic necrotic lesion, which was treated with laparoscopic necrosectomy.

Two months later, we performed an Octreoscan and serum tests of vasoactive intestinal peptide (VIP) and chromogranin A (CgA), which were normal. EUS with contrast enhanced detected areas of fibrosis [3]. At present, after a follow-up period of 18 months, the patient is asymptomatic, without recurrent disease; abdominal nuclear magnetic resonance is negative.

EUS is particularly useful in the assessment of small pancreatic tumors missed by other imaging techniques [3-5]. EUSFNA [1] is a gold standard in the management of pancreatic tumors. Unusually, we have explored a new field of EUS that permits injection of drugs directly into the tumor. In our experience, if surgical therapy is refused by the patient or contraindicated because of poor general health conditions, EUS-guided injection is a safe and effective treatment in endocrine lesions, which can eliminate hormonal alterations.

It has been demonstrated that ethanol injection is a safe technique for the treatment of hepatic and thyroid lesions [6]. In our case we have used the same technique to treat pancreatic lesions, thus avoiding surgery, which has high morbidity and mortality; the duration of hospitalization was also reduced. A multicenter study is necessary to establish a standardized procedure for EUS-ethanol injection in neuroendocrine pancreatic tumors.

Endoscopy_UCTN_Code_CPL_1AL_2AD 
N. Muscatiello' ${ }^{1}$, A. Salcuni ${ }^{2}$,

L. Macarini ${ }^{3}$, M. Cignarelli ${ }^{2}$, S. Prencipe ${ }^{1}$, M. di Maso ${ }^{1}$, M. Castriota ${ }^{4}$,

V. D’Agnessa ${ }^{4}$, E. lerardi ${ }^{1}$

1 Gastroenterology Unit, Ospedali Riuniti, Foggia, Italy

2 Endocrinology Unit, Ospedali Riuniti, Foggia, Italy

3 Radiology Unit, Ospedali Riuniti, Foggia, Italy

${ }^{4}$ Pathology Unit, Ospedali Riuniti, Foggia, Italy

\section{References}

1 Gress F, Gottlieb K, Sherman S, Lehman G. Endoscopic ultrasonography-guided fine needle aspiration biopsy of suspected pancreatic cancer. Ann Intern Med 2001; 134 459-464

2 Matthes K, Mino-Kenudson M, Sahani DV et al. Concentration-dependent ablation of pancreatic tissue by EUS-guided ethanol injection. Gastrointest Endosc 2007; 65: 272 277

3 Becker D, Strobel D, Bernatik T, Hahn EG. Echo-enhanced color and power-Doppler EUS for the discrimination between focal pancreatitis and pancreatic carcinoma. Gastrointest Endoscopy 2001; 53: 784-789

4 Ichikawa T, Peterson MS, Federle MP et al. Islet cell tumor of the pancreas: biphasic CT versus MR imaging in tumor detection. Radiology 2000; 216: $163-171$

5 Anderson MA, Carpenter S, Thompson NW et al. EUS is highly accurate and directs management in patients with neuroendocrine tumors of the pancreas. Am J Gastr 2000; 95: $2271-2277$

6 Livraghi T, Giorgio A, Marin G et al. Hepatocellular carcinoma and cirrhosis in $746 \mathrm{pt}$ : long-term results of percutaneous ethanol injection. Radiology 1995; 197: 101 - 108
Bibliography

DOI 10.1055/s-2007-966962

Endoscopy 2007; 39: E258-E259

(C) Georg Thieme Verlag KG Stuttgart · New York . ISSN 0013-726X

\section{Corresponding author}

\section{N. Muscatiello, MD}

Gastroenterology Unit Ospedali Riuniti

Viale Luigi Pinto, 1 71100 Foggia

Italy

Fax: $+39-881733870$

nmuscatiello@libero.it 